\title{
corrosión de armaduras en los hormigones armados y pretensados
}

J. CALLEJA*

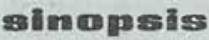

Dentro del capítulo general de la «durabilidad» o resistencia química en el tiempo de los conglomerados hidráulicos (morteros $\mathrm{y}$ hormigones armados $\mathrm{y}$ pretensados), el apartado relativo a la «corrosión por su importancia técnica y económica.

Esta importancia va siendo reconocida cada día más, a pesar de ser (o de haber sido) el de la corrosión uno de los temas menos familiares en el ámbito de la construcción. La razón estriba en la fuerza de los hechos, y se maniflesta con claridad en las tareas encomendadas y en los problemas presentados a los laboratorios de investigación y ensayos de materiales.

La corrosión es un fenómeno físicoquímico que cae dentro del dominio de la ELECTROQUIMICA y que debe estudiarse y enjuiciarse con arreglo a las leyes fundamentales de esta disciplina. Lo mismo cabe decir por lo que respecta a los métodos de eprotección» contra la corrosión.

La corrosión es un «riesgo" aleatorio que se corre y contra el que hay que prevenirse mediante un «seguro», representado por dichas medidas de protección.

El remedio «más gácil» desde un punto de vista «técnico», y «más barato» desde un punto de vista «económico», es siempre de carácter apreventivo» antes que «curativo», es decir, es el tomado ya en la etapa de proyecto de la obra o estructura de que se trate.

En el caso del hormigón, la mejor protección de las armaduras la da, en primer lugar, la aplicación de un «código de buena prácticas a la elaboración del material y a la ejecución de la obra, $y$, en segundo lugar, la observancia de los más elementales principios de la Electroquímica en el aspecto de la corrosión.

La utilización de aditivos acelerantes de fraguado y endurecimiento con naturaleza «clorurada” y el estado de «tensión» de las armaduras son aspectos que merecen especial consideración, así como las posibles acciones electroquímicas debidas a *corrientes

\section{Fuchoros

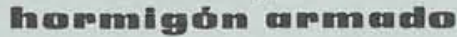

Como ya se indicó, las interacciones entre el hormigón y sus armaduras de acero condicionan la resistencia de éstas a la corrosión, y están a su vez determinadas por las características del acero, por las del hormigón y por las del medio ambiente.

Constituyen o pueden constituir medios electrolíticos agresivos externos los siguientes:

I) los suelos más o menos salinos;

II) el agua del mar;

III) las aguas naturales;

IV) los desagüies industriales;

V) las diversas sustancias contenidas en depósitos de hormigón o transportadas por conducciones de este material;

VI) la atmósfera húmeda, contaminada o no por agentes agresivos; etc.

Por otra parte, la alcalinidad y los compuestos del cemento fraguado y, eventualmente, las adiciones químicas añadidas al hormigón constituyen el medio electrolítico directo $o$ inmediato en el que las armaduras se encuentran.

- Doctor en Ciencias Químicas, Investigador Jefe del Departamento de Química del Instituto Eduardo Torroja de la Construcción y del Cemento, Patronato "Juan de la Cierva" de Investigación Científica y Técnica, Consejo Superior de Investigaciones Científicas. 
El acero de las armaduras puede contener como elementos secundarios carbono, azufre, silicio, manganeso, etc., y, además, escamas de laminación (el acero laminado en caliente), las cuales forman recubrimientos discontinuos y herrumbre. Otras alteraciones son debidas a los tratamientos o a las condiciones de almacenamiento y conservación de los aceros. Todas estas circunstancias, por suponer heterogeneidades, coadyuvan a la formación de microelementos galvánicos de corrosión. Otras heterogeneidades en la superficie de las armaduras pueden ser debidas a distribución irregular de los áridos o de la pasta conglomerante, lo que origina huecos; a juntas de hormigonado deficientes; a empleo de conglomerantes distintos en una misma estructura o en parte de la misma; a la existencia o formación de fisuras; etc.

El hormigón puede ser irregularmente poroso y permeable, y presentar zonas con distinta accesibilidad para la humedad y los agresivos electrolíticos eventuales. La porosidad y la permeabilidad dependen de algunos de los factores acabados de señalar y, además y principalmente, de los siguientes:

I) de la naturaleza y granulometría de los áridos;

II) de la dosificación de cemento (relación cemento/áridos);

III) de la cantidad de agua de amasado y de la relación agua/cemento;

IV) del sistema de puesta en obra (picado, apisonado, vibrado, proyectado, centrifugado, etc.);

V) de las condiciones de curado; etc.

Aun siendo uniformemente poroso, el hormigón puede tener partes enterradas o sumergidas, difícilmente accesibles al aire, y partes exteriores más fácilmente accesibles al mismo (pilotes). Si está totalmente enterrado (conducciones, tuberías, galerías), la parte inferior y más profunda es siempre más difícilmente accesible al aire que la superior. El terreno en que se encuentre el hormigón puede ser más o menos permeable al aire, según su condición arcillosa o arenosa en cada caso. Exterior, sumergido o enterrado, y aun siendo de porosidad uniforme, el hormigón puede tener armaduras más o menos superficiales, es decir, revestidas por un recubrimiento de material pétreo de espesor mayor o menor. En todos estos casos pueden crearse elementos o pilas de aireación diferencial.

El hormigón puede contener en su masa sales, y concretamente cloruros (sódico o cálcico), como consecuencia de utilizar estas sales para eliminar el hielo (cloruro sódico o cálcico) en el caso de pistas; como consecuencia de adiciones hechas al hormigón en fresco para acelerar su fraguado y endurecimiento o para mejorar su resistencia a las heladas (cloruro cálcico), o como resultado de emplear como áridos arenas de playa no lavadas (cloruros alcalinos y cloruro magnésico). En tales casos estas sales, por una desigual distribución atribuible a causas diversas, pueden dar lugar a pilas de concentración. Al mismo tiempo aumentan la conductividad del hormigón y contribuyen con ello a producir o a acelerar la corrosión.

El hormigón puede verse sometido a un proceso electrolítico por paso de corrientes vagabundas o derivadas a través de sus armaduras, siendo facilitado dicho paso e intensificados sus efectos por la presencia o concurrencia de alguna o algunas de las circunstancias señaladas y, sobre todo, por la acción de las sales solubles.

\subsection{Corrosión de armaduras producida o acelerada por la presencia de cloruros en el hormigón}

Acaba de ser mencionado uno de los casos más típicos en que puede darse este tipo de corrosión: la adición de cloruros al hormigón fresco como aceleradores de su fraguado y endurecimiento, o al hormigón endurecido para eliminar el hielo. A esto hay que añadir que el cloruro cálcico forma parte de la mayoría de los aditivos (aireantes, 
fluidificantes, etc.) que se añaden al hormigón, los cuales de por sí suelen retrasar el fraguado; el cloruro cálcico contrarresta esta acción retardadora*.

También entran en este caso las acciones del agua del mar y de las atmósferas marinas sobre el hormigón armado.

\subsubsection{Corrosión del hierro en presencia de cloruros}

Como se indicaba en el punto 3, los aniones salinos, y en particular el anión cloro de los cloruros cálcico o sódico, pueden actuar en la zona catódica protegida, desplazando el potencial hacia valores activos y convirtiéndola en anódica, susceptible de corrosión.

En efecto, en presencia de cloruro sódico o de cloruro cálcico la reacción catódica (XX) -véase 2.5-que supone un aumento del $\mathrm{pH}$, va acompañada de un alejamiento de los aniones $\mathrm{Cl}^{-}$que se desplazan hacia la región anódica; en contrapartida, en la zona catódica se acumulan cationes sodio $\mathrm{Na}^{+}$o calcio $\mathrm{Ca}^{2+}$. Así, pues, los productos de corrosión serán en la zona anódica cloruro ferroso y en la catódica hidróxido sódico (o cálcico), ambos solubles, ninguno de los cuales puede, en consecuencia, impedir ni retardar el proceso anódico ni el catódico, ni proteger al hierro de la corrosión.

Por difusión a través del electrólito los iones ferrosos $\mathrm{Fe}^{2+}$ y los iones hidroxilo $\mathrm{OH}^{-}$ pueden reaccionar lejos de las respectivas zonas anódica y catódica, dando lugar a la formación de hidróxido ferroso $\mathrm{Fe}(\mathrm{OH})_{2}$, el cual, por la acción del oxígeno del aire, puede transformarse en hidróxido férrico $\mathrm{Fe}(\mathrm{OH})_{3}$, en óxido férrico hidratado $\mathrm{Fe}_{2} \mathrm{O}_{3}$. - $\mathrm{H}_{2} \mathrm{O}$, o bien en un oxi-hidróxido de hierro también hidratado $\mathrm{FeO}(\mathrm{OH}) \cdot \mathrm{H}_{2} \mathrm{O}$.

Estos productos, o sus mezclas en diversas proporciones según las circunstancias, constituyen la llamada herrumbre, que es insoluble. Pero, por formarse y depositarse lejos de las zonas anódica y catódica (electrodos del elemento galvánico local), tampoco pueden evitar ni atenuar los respectivos procesos ni, por consiguiente, impedir la corrosión.

Las reacciones descritas están puestas de manifiesto en el gráfico esquemático de la figura 9, que representa el mecanismo de la acción corrosiva de una gota de disolución salina (cloruro) sobre una lámina de hierro. A este gráfico corresponde el de la figura 10, que representa la acción corrosiva de una gota de agua sobre la misma lámina. En este caso la acción corrosiva es meramente debida a una aireación diferencial entre la zona periférica y la parte central de la gota, mientras que en el caso de la figura 9, a este efecto se suma el efecto salino, por lo que la corrosión es mucho mayor.

Con mayor o menor intensidad en cada caso, en el lugar ocupado por cada gota se tiene el resultado que muestra el esquema de la figura 11, con un cráter de corrosión y un depósito anular de herrumbre. Este efecto repetido sobre diversos puntos de una chapa de hierro da lugar a la figura esquemática de corrosión puesta de manifiesto en la figura 12.

Aun suponiendo que la herrumbre se formase directamente sobre la zona anódica o sobre la catódica, tampoco podría impedir ninguno de los dos procesos ni, por lo tanto, la corrosión. La causa estriba en que los productos que forman la herrumbre son muy voluminosos y porosos, ya que su volumen es mayor que la suma de los volúmenes de las sustancias a partir de las cuales resultan, por lo cual su formación tiene lugar con carácter expansivo y no pueden constituir un recubrimiento impenetrable para los iones. Por el contrario, éstos se difunden a través de la herrumbre con gran facilidad, por lo que el proceso corrosivo continúa.

Por otra parte, y según lo acabado de exponer, la formación de herrumbre transcurre con un notorio aumento de volumen, y cuando se ve confinada da lugar a unas fuerzas

* "Aditivos para el hormigón», I.E.T.c.c., próxima publicación. J. Calleja y B. Bacle. 


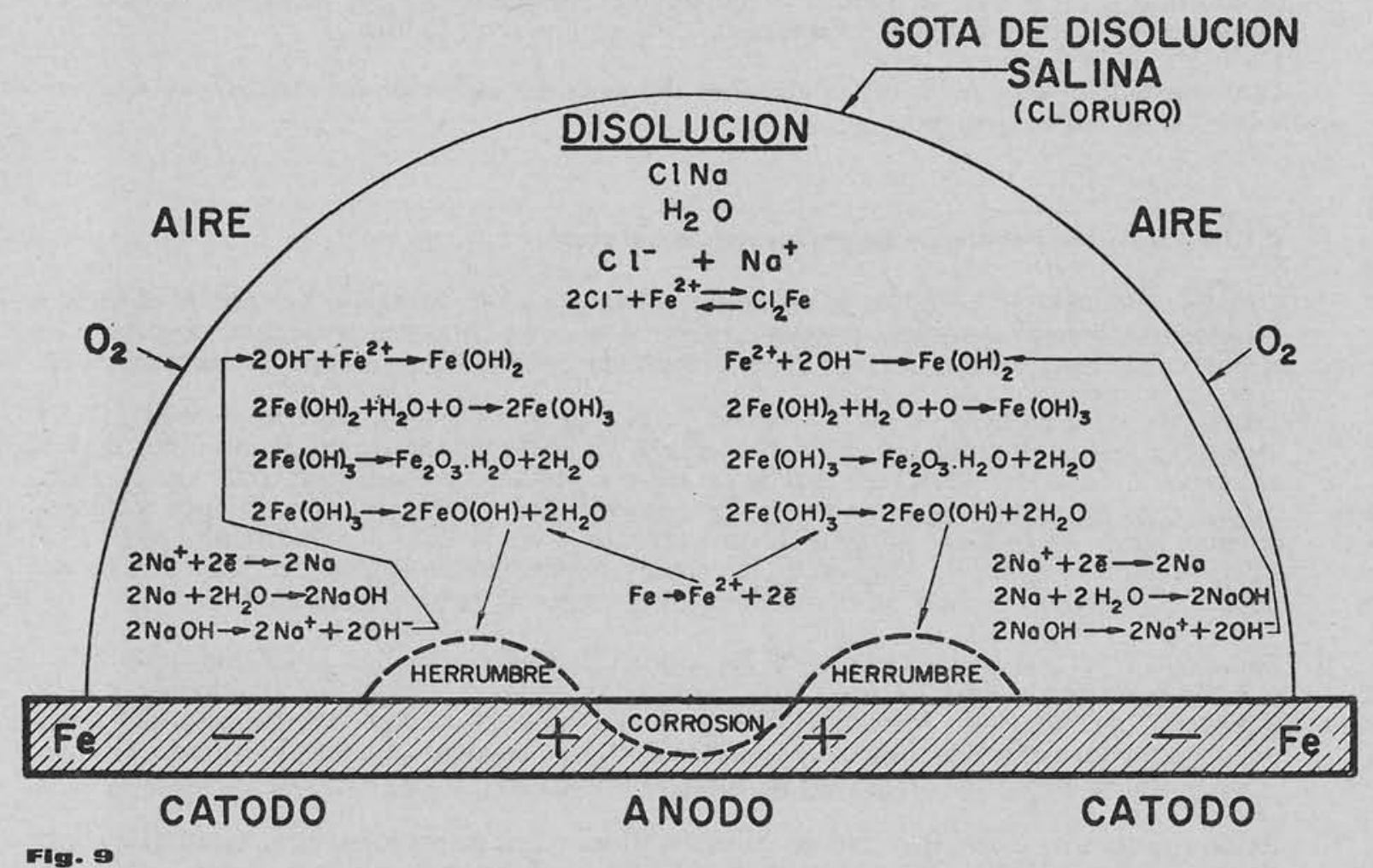

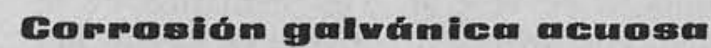

(Aireación diferencial)

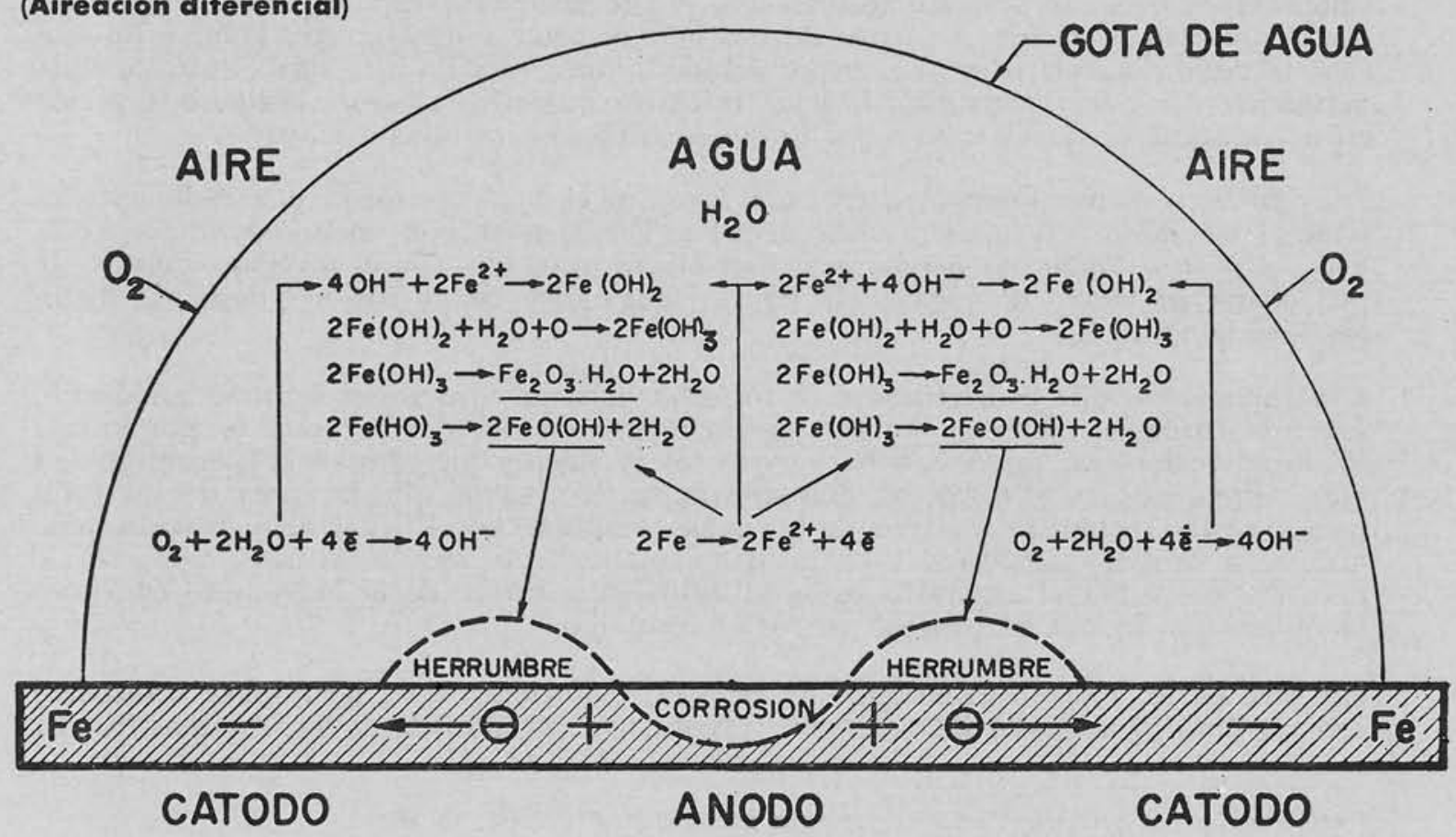




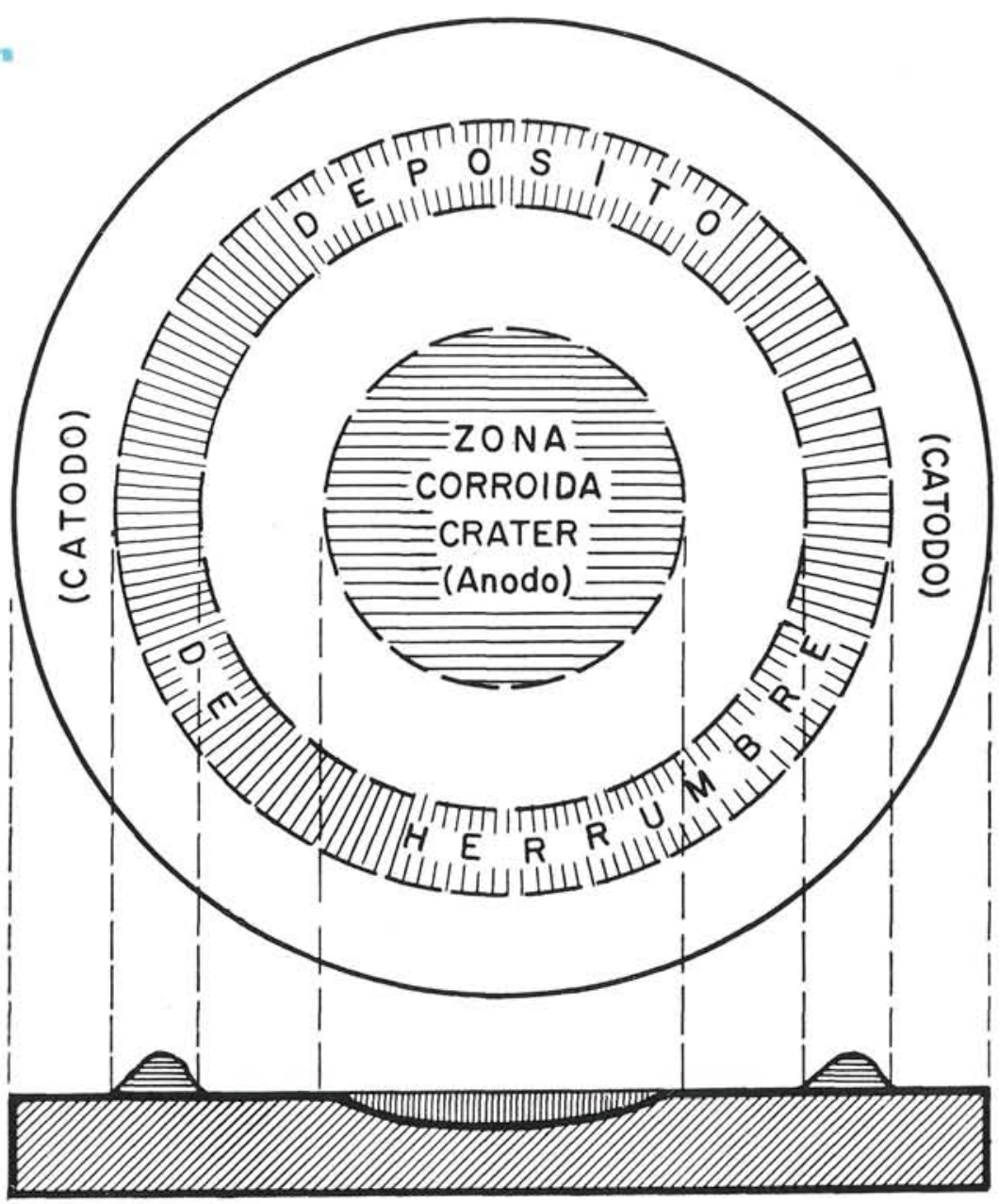

aspecto the unn

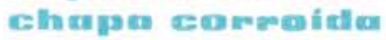

Fig. 12

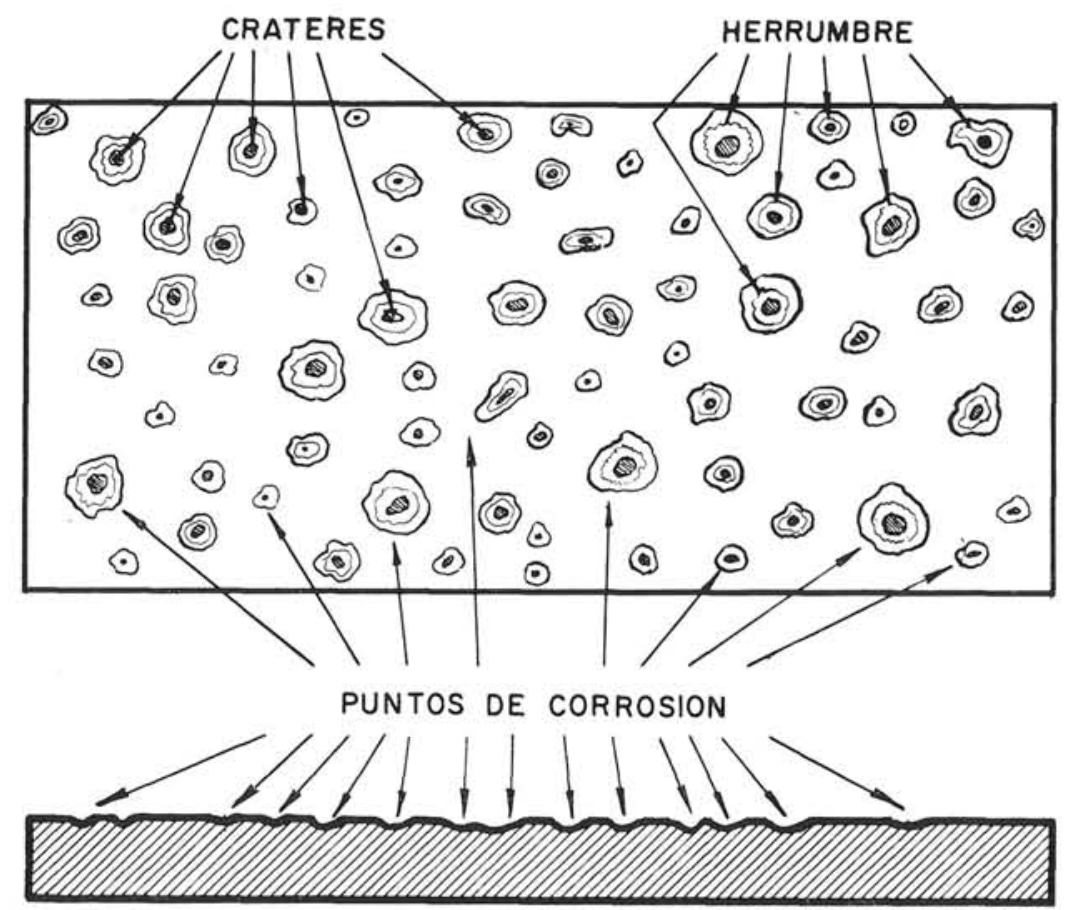




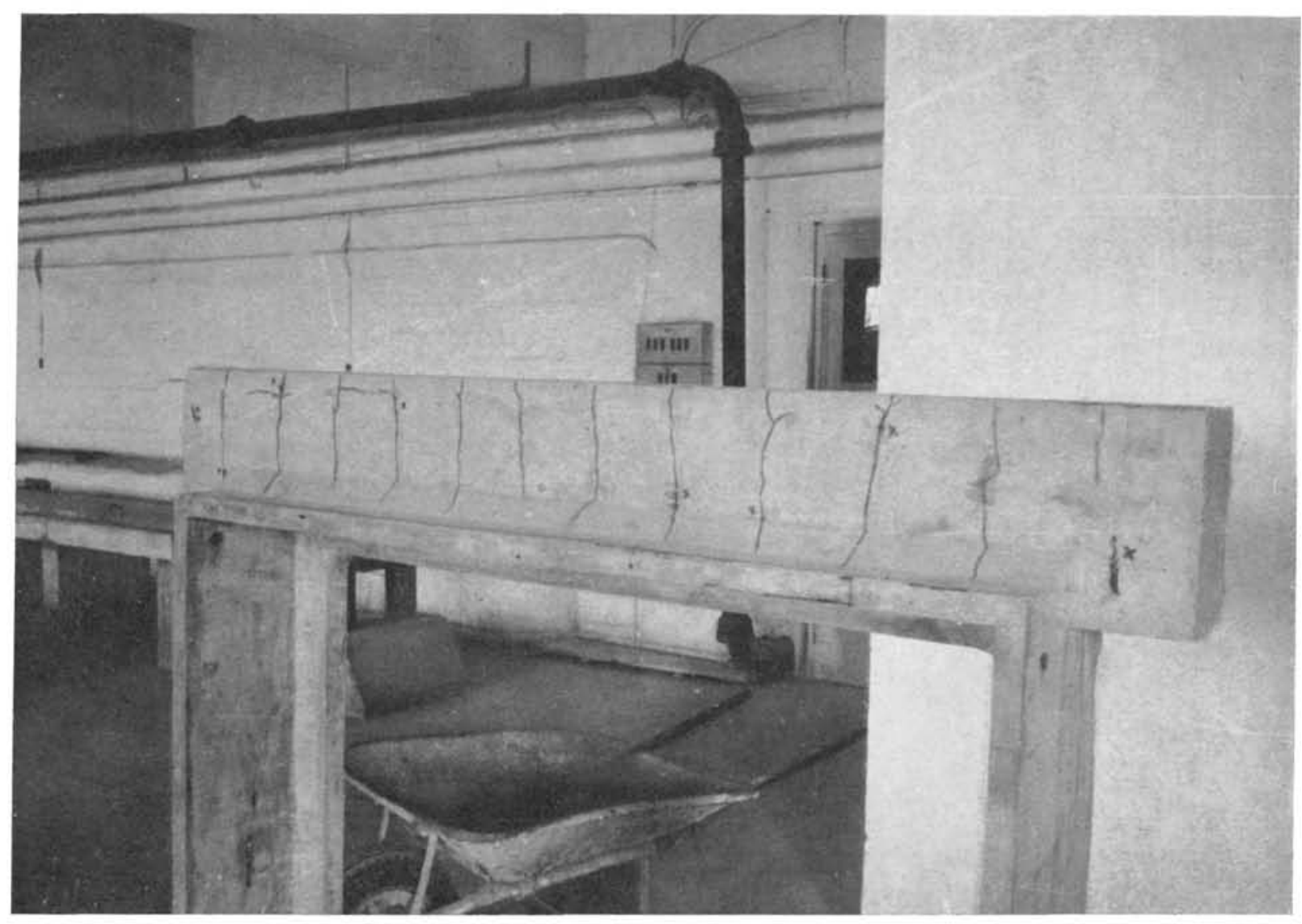

Fing - 13

expansivas muy considerables. En el caso de las armaduras del hormigón corroídas, estas fuerzas pueden llegar a producir fisuras y grietas en el material pétreo, debilitando su resistencia mecánica y provocando una disminución de la adherencia entre el hormigón y las armaduras, lo que a su vez origina un nuevo debilitamiento y abre nuevos cauces a la corrosión.

Es fácil apreciar en hormigones armados expuestos a atmósferas marítimas severas, o en hormigones que han sido amasados con una disolución de cloruro cálcico, que afloran manchas de herrumbre a la superficie de los mismos. Esta herrumbre sale a través de microfisuras que después se hacen perceptibles, las cuales son ocasionadas por la fuerza expansiva del óxido hidratado formado y coinciden en posición, bien sea con las propias armaduras, o bien con los estribos de éstas (fig. 13). Las fisuras pueden continuar agrandándose hasta provocar la rotura del hormigón y dejar las armaduras al descubierto. A veces no se observan manchas exteriores de herrumbre, pero los efectos son idénticos.

De todo esto se deduce que la corrosión electroquímica es mucho más frecuente y nociva que el ataque químico directo, aunque el resultado final en cuanto a la transformación experimentada por el metal sea el mismo. Hay que tener en cuenta también que la conductividad eléctrica del hormigón, que tampoco tiene un valor definido, ya que depende de su contenido de agua libre mucho más que de la edad del material, de la que prácticamente es independiente, aumenta de manera notable con la presencia de sales (cloruro cálcico) y polvos de sustancias más conductoras que los áridos. 


\subsubsection{Cloruro cálcico y cemento portland}

Antes de tratar de la acción del cloruro cálcico sobre las armaduras conviene considerar la que ejerce sobre el propio hormigón o, mejor, sobre el cemento.

Uno de los constituyentes principales del clinker de cemento portland es el aluminato tricálcico: $3 \mathrm{CaO} \cdot \mathrm{Al}_{2} \mathrm{O}_{3}=\mathrm{C}_{3} \mathrm{~A}$, que reacciona muy rápidamente con el agua y es el responsable de que en alguna circunstancia se dé el indeseado fenómeno del fraguado rápido. Para evitar éste, al moler el clínker en las fábricas de cemento se le añade yeso para convertirlo precisamente en cemento.

La gran tendencia del $\mathrm{C}_{3} \mathrm{~A}$ a hidratarse rápidamente se ve contenida por la formación, en presencia de sulfato cálcico y en el medio alcalino fuerte (saturado de hidróxido cálcico $\mathrm{Ca}(\mathrm{OH})_{2} ; \mathrm{pH}=12$ ) de la pasta de cemento, de sales complejas de escasa solubilidad: los sulfoaluminatos cálcicos $3 \mathrm{CaO} \cdot \mathrm{Al}_{2} \mathrm{O}_{3} \cdot 3 \mathrm{SO}_{4} \mathrm{Ca} \cdot 32 \mathrm{H}_{2} \mathrm{O}$ (trisulfato) y $3 \mathrm{CaO}$. - $\mathrm{Al}_{2} \mathrm{O}_{3} \cdot \mathrm{SO}_{4} \mathrm{Ca} \cdot 10-12 \mathrm{H}_{2} \mathrm{O}$ (monosulfato) formulados abreviadamente por $\mathrm{C}_{3} \mathrm{ACs}_{3} \mathrm{H}_{30}$ y $\mathrm{C}_{3} \mathrm{ACsH}_{12}$, respectivamente. Estos sulfoaluminatos protegen y aislan al $\mathrm{C}_{3} \mathrm{~A}$, retardando su reacción con el agua y haciendo que el fraguado sea normal en vez de rápido. De aquí que todos los cementos de tipo portland contengan sulfato cálcico (preferentemente yeso dihidrato, o anhidrita, o mezcla de ambos) como retardador o regulador del fraguado.

Para el contenido de yeso de los cementos se establecen en las Normas relativas a los materiales conglomerantes, máximos, pero no mínimos, siendo así que cada clínker, según su contenido de $\mathrm{C}_{3} \mathrm{~A}$ principalmente, requeriría un óptimo de yeso. Esto hace que, en general, los cementos tengan contenidos de yeso inferiores a los óptimos.

El cloruro cálcico $\mathrm{Cl}_{2} \mathrm{Ca}$ forma con el $\mathrm{C}_{3} \mathrm{~A}$ complejos análogos a los del sulfato cálcico, y precisamente el cloroaluminato cálcico de fórmula $3 \mathrm{CaO} \cdot \mathrm{Al}_{2} \mathrm{O}_{3} \cdot \mathrm{Cl}_{2} \mathrm{Ca} \cdot 10 \mathrm{H}_{2} \mathrm{O}(\mathrm{mo}$ noclorado: $\mathrm{C}_{3} \mathrm{AClH}_{10}$ ). De los dos tipos de complejos teóricamente se forman antes y más fácilmente los sulfoaluminatos, por su mayor insolubilidad y estabilidad. Una vez formados, el $\mathrm{C}_{3} \mathrm{~A}$ restante o disponible es el que puede reaccionar fijando cloruro cálcico para formar el cloroaluminato. A su vez, cuando se ha formado todo el cloroaluminato posible, el cloruro cálcico restante, si lo hay, es el que queda libre y disponible a efectos corrosivos. No así el fijado en forma de sal compleja, a menos que ésta se desdoble por alguna causa. En consecuencia, no hay que olvidar que, en este aspecto, el cloroaluminato cálcico complejo hace de reserva potencial de iones cloro, y que si dicha reserva potencial se actualiza y pone en acción, a partir de ese momento la corrosión de las armaduras puede producirse o acelerarse.

En definitiva, la corrosión de las armaduras por la presencia de cloruro cálcico en el hormigón no depende sólo de la cuantía o concentración de esta sal, sino más bien de la cuantía o concentración de la sal que queda libre sin transformarse en el complejo cloroaluminato.

La cuantía o concentración de sal libre dependerá, además:

I) de la cantidad de cloruro cálcico añadida al hormigón;

II) de la cantidad de cemento que tenga el hormigón;

III) del tipo de cemento;

IV) de la cantidad de aluminato tricálcico que tenga el cemento;

V) de la cantidad de yeso que tenga el cemento.

Los dos primeros factores relativos al hormigón se suelen tener en cuenta siempre, ya que constituyen en realidad uno solo, y las dosis de cloruro cálcico añadidas en concepto de acelerador del fraguado y del endurecimiento se especifican y expresan en tanto por ciento respecto del peso del cemento. 
Los tres últimos factores, en cambio, no se tienen en cuenta nunca, porque no se suele conocer ni determinar el contenido de yeso de los cementos, y mucho menos el contenido de aluminato tricálcico, si bien a partir de un análisis químico ordinario el primero se conocería, y el segundo podría calcularse con una aproximación aceptable. Por otra parte, en muchos casos no se hace distinción entre cementos portland, siderúrgicos o puzolánicos.

\subsubsection{Cloruro cálcico y corrosión de armaduras}

Tal vez en lo anteriormente expuesto resida la explicación de la falta de concordancia entre los limites que los distintos investigadores fijan para las dosis de cloruro cálcico tolerables a efectos de corrosión de armaduras, e incluso entre los resultados cualitativos de los correspondientes estudios.

Las consideraciones anteriores se ven refrenadas por multitud de hechos experimentales comprobados, entre los cuales cabe destacar los siguientes:

I) la mayor parte del cloruro cálcico añadido al hormigón usualmente (2 por 100 de un cloruro cálcico comercial de 70 por 100 de riqueza en sal anhidra, es decir, un 1,4 por 100 de sal anhidra) queda combinado en forma de complejo en el cemento hidratado, si bien en la disolución queda aún una parte sin combinar;

II) la cantidad de cloruro cálcico que queda libre es tanto mayor (a constancia de todo lo demás) cuanto mayor es la cantidad inicialmente añadida;

III) a igualdad de todo lo demás, con cementos resistentes a sulfatos (de contenido bajo de aluminato tricálcico) la concentración de cloruro cálcico libre es mayor que con cementos portland (de contenido mayor de aluminato tricálcico). Por razones de dilución los cementos siderúrgicos y los puzolánicos contienen inicialmente menos aluminato tricálcico que los portland, y por ello contribuyen a dar una concentración inicial de cloruro cálcico mayor;

IV) la cantidad de cloruro cálcico libre es tanto mayor cuanto menores son los contenidos de óxido alumínico y óxido férrico en conjunto del cemento;

V) la cantidad de cloruro cálcico libre es tanto mayor cuanto menor es la relación óxido alumínico/óxido férrico;

VI) la cantidad de cloruro cálcico libre es tanto mayor cuanto mayor es el contenido de yeso del cemento;

VII) la cantidad de cloruro cálcico libre es tanto mayor cuanto mayor es el contenido de álcalis libres del cemento;

VIII) la cantidad de cloruro cálcico libre es tanto mayor cuanto mayor es la temperatura, ya que en tales condiciones son más estables los aluminatos hidratados exentos de cloruro cálcico;

IX) la concentración de cloruro cálcico libre es tanto mayor cuanto menor es la relación agua/cemento;

X) cuanto mayor es la cantidad o concentración de cloruro cálcico libre, tanto mayor es la corrosión producida.

Estos hechos, a su vez, permiten establecer una serie de conclusiones generales, a saber:

el cloruro cálcico en pequeñas dosis (hasta 2 por 100 de producto comercial con riqueza de 70 a 80 por 100 de sal anhidra, correspondiente a 1,4 ó 1,6 por 100 de cloruro cálcico anhidro, respecto del peso del cemento) se suele emplear sin gran probabilidad de corrosión a cualquier edad, con cemento port- 
land ordinario sometido a un curado ordinario. Con estas mismas dosis hay una gran probabilidad de tener una ligera corrosión con cementos resistentes a sulfatos (portland de bajo contenido de aluminato tricálcico: tipo «PAS " según las Normas españolas P.C.C.H. 64 del I.E.T.c.c.), con cementos siderúrgicos o con el mismo portland sometido a un curado térmico. Hay también una mayor probabilidad de corrosión con curado húmedo que con curado seco, sobre todo a corto plazo;

con dosis superiores a 2 por 100 de cloruro cálcico siempre existirá una probabilidad considerable de tener corrosión en cualquier caso y con cualquier cemento;

con dosis inferiores a 2 por 100 aún existe una probabilidad, aunque mucho menor, de tener corrosión, y precisamente más en los casos más favorables para ello, lo que indica que no hay un umbral en la cantidad o concentración del cloruro cálcico, por debajo del cual no se pueda dar la corrosión. Es decir, posibilidad de corrosión la hay siempre, y tan sólo la probabilidad de la misma es la que varía, según las circunstancias;

el cloruro cálcico en cualquier caso puede aumentar y acelerar la corrosión natural, al menos potencialmente, y así, debe entenderse que, siempre que haya cloruro cálcico en el hormigón, la corrosión de las armaduras, a igualdad de todo lo demás, es mayor (en el mejor de los casos igual, pero nunca menor) que cuando no lo hay;

en la acción corrosiva del cloruro cálcico, sobre todo por lo que se refiere a los ensayos e investigaciones de laboratorio, hay que tener en cuenta el plazo o tiempo al que la corrosión se manifiesta con una cierta y determinada intensidad, a constancia de todo lo demás. No se puede decir, mientras no se tenga seguridad absoluta de ello (caso excepcional) que no habrá corrosión, o que ésta no pasará de un cierto valor en unas condiciones dadas; es preferible y más correcto referir tales afirmaciones al plazo a que se hayan hecho las correspondientes observaciones. En todo caso es mejor hablar siempre de probabilidad de corrosión, debiendo entenderse que ésta rara vez es nula;

no debe olvidarse que al tratar del efecto de las dosis de cloruro cálcico en la corrosión de las armaduras no se han mencionado en los puntos anteriores las características del hormigón ni las del medio ambiente (lo cual se hace más adelante), pues a veces las armaduras de un buen hormigón con cloruro cálcico no se corroen o se corroen menos que las de otro hormigón malo con menos cloruro cálcico, o incluso sin cloruro cálcico alguno. De aquí que muchas de las consideraciones anteriores se hayan basado en la igualdad o constancia «de todo lo demás». Por lo que respecta a las características y ejecución del hormigón desde el punto de vista de la corrosión, tanto del material pétreo como de las armaduras, deben observarse siempre las que pudieran llamarse normas de un código de buena práctica.

\section{ธ.}

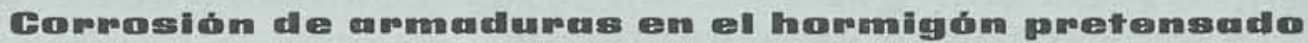

La corrosión de alambres en el hormigón pretensado no es frecuente en circunstancias normales, por el consabido hecho de que el hormigón, y más concretamente la pasta de cemento, constituye una buena protección para el acero, y porque el hormigón pretensado suele ser un material de proyecto, ejecución y vigilancia muy cuidados.

Claro está que en otras circunstancias puede haber corrosión y entonces ésta es, a la vez, más fácil y rápida, y más grave que en el hormigón armado. Más fácil y rápida, porque los alambres sometidos a tensión son más susceptibles y pueden combinar dicha tensión con la corrosión ordinaria, dando lugar a tipos mixtos de corrosión, de evo- 
lución más acelerada. Más grave, porque, dada la pequeña sección de los alambres, moderadas corrosiones que serían inocuas en el caso del hormigón armado con redondos gruesos, en el caso del pretensado pueden ocasionar disminuciones de sección tales que provoquen la rotura de las armaduras.

Que esto es así lo prueba el hecho de que en secciones de tuberías de hormigón pretensado, con armaduras longitudinales sin tensar y armadura helicoidal tensada, ésta se corroe mucho más que aquéllas.

Así, pues, cualquier causa que en el hormigón armado pueda promover un efecto corrosivo tolerable, en el hormigón pretensado puede ocasionar un verdadero desastre. Entre tales causas se cuenta, por supuesto, la presencia de cloruro cálcico.

La corrosión en estos casos se produce en zonas de la armadura coincidentes con huecos, cuya dimensión es a veces del orden del diámetro de los alambres. Ni que decir tiene que en tales circunstancias el empleo de cloruro cálcico (o, en general, un ataque por cloruros) puede ser catastrófico y, en consecuencia, dicho empleo debe ser evitado.

Como ejemplo aleccionador se cita y detalla en la bibliografía el caso de la conducción de aguas de Regina, en Saskatchewan (Canadá), hecha de tubos de hormigón pretensado empalmados. Cada sección de tubería constaba de un núcleo de hormigón con armaduras longitudinales, sobre el que se arrollaba una armadura helicoidal pretensada, recubierta exteriormente de hormigón proyectado ("gunita»). Este contenía hasta 5 por 100 de cloruro cálcico. A ello unía una serie de defectos y circunstancias propicias a la corrosión, tales como el ser distintos los cementos del núcleo de hormigón y de la capa de hormigón proyectado; el tener las armaduras menor recubrimiento en los extremos de los tubos, y el estar la tubería sometida exteriormente y en su parte inferior a la acción de aguas agresivas. La corrosión se presentó pronto y con carácter demoledor, manifestándose de preferencia en la parte inferior (e interna) de la armadura helicoidal, en lugares coincidentes con huecos debidos a deficiente recubrimiento y adherencia, en los que fue muy apreciable la concentración de iones cloro y muy grande la probabilidad de que el hormigón proyectado no rodease por completo a los alambres.

Como dato curioso pudo observarse que los tubos almacenados en el parque, es decir, los no puestos aún en servicio, ni siquiera en obra, también se destruyeron. La causa en este caso fue la recirculación del agua de las balsas de curado, la cual llegó a contener 2 gramos de sulfato cálcico y 1,4 gramos de cloruro cálcico por litro.

Es de apreciar que todo esto constituye un ejemplo típico y hasta exagerado, no muy frecuente, por fortuna, de código de mala (pésima) práctica.

Como consecuencia de estos resultados y de otros muchos análogos, es una medida prudente prescindir en absoluto del empleo de cloruro cálcico como acelerador del endurecimiento en el caso del hormigón pretensado.

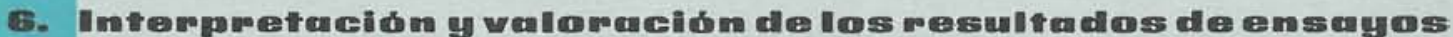

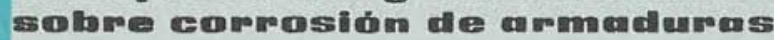

A consecuencia de la volubilidad e inestabilidad de los potenciales del hierro y de su dependencia (así como la de la conductividad eléctrica del hormigón) de tan complejo y amplio grupo de variables, las conclusiones de las distintas investigaciones llevadas a cabo en este campo son a veces contradictorias, y los resultados a que llegan diferentes laboratorios están con frecuencia en desacuerdo entre sí y con los observados en la práctica.

Esto obliga a que todo estudio, sea de laboratorio o a escala real de obra, deba recaer sobre un número suficientemente grande de series de probetas idénticas, para poder so- 
meter los resultados a un análisis estadístico, si se quiere disponer de elementos de juicio sanos para poder determinar la probabilidad de corrosión.

También esto induce a considerar inútiles en muchos casos los ensayos a largo plazo, e inadecuados los tratamientos acelerados que falsean los resultados. Tan sólo estos tratamientos pueden tener algún sentido si son lo suficientemente drásticos como para situar los resultados en el lado de la seguridad, es decir, si se puede afirmar, a la vista de un resultado favorable, que nunca habrá corrosión, aunque en el caso de un resultado desfavorable pueda no haberla.

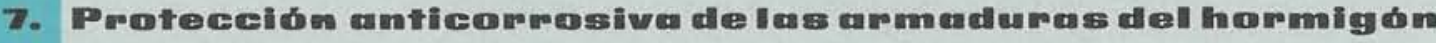

\subsection{Consideraciones generales sobre la protección anticorrosiva}

Se ha considerado con razón la protección anticorrosiva como un sistema de seguro contra un riesgo: el de la corrosión. La mayor compacidad del hormigón, la más alta dosificación del mismo, el mayor espesor de los recubrimientos y cuantas medidas de precaución se adopten, hasta llegar a una verdadera y compleja instalación de protección, pueden no servir a ninguna finalidad mecánica ni estructural del proyecto, pero tienen el sentido de una prima de seguro que se abona contra unas posibles contingencias, y el de una medicina de tratamiento preventivo.

Las contingencias, cuando se presentan, suponen, en capital o en anualidades, un valor (el que correspondería al tratamiento curativo) muy superior al abonado "al seguro". Por otra parte, la "prima» a pagar es tanto más reducida cuanto más pronto se hace el seguro, y mínima si éste "se concierta" al comienzo de la obra, ya que entonces, al no haber pies forzados, las posibilidades son máximas y las dificultades mínimas.

Se comprende lo difícil que es dar con carácter absoluto una serie de recomendaciones encaminadas a evitar un problema tan complejo como el de la corrosión de las armaduras del hormigón. Las que se den sólo podrán tener un valor relativo y, de acuerdo con esto, se exponen en lo que sigue los remedios preventivos más unánimemente reconocidos como eficaces, los cuales son también los más interesantes, sin que el orden de exposición implique siempre y necesariamente orden de preferencia.

En primer lugar, debe evitarse al máximo el empleo de cloruro cálcico u otras sales o electrólitos análogos, así como el de los productos de adición que los contengan, puesto que en ambos casos hay grandes probabilidades de que tales prácticas provoquen o aceleren la corrosión. Téngase en cuenta que no se ha llegado a poder establecer un umbral de contenido o concentración de cloruro cálcico, por debajo del cual no se produzca corrosión a plazo más o menos largo. El cloruro cálcico, aun en dosis pequeñas, puede ser un enemigo en potencia.

En segundo lugar, hacer un hormigón armado o pretensado cuyas armaduras estén protegidas al máximo, tanto si contiene como si no contiene cloruro cálcico. Puesto que los remedios están en íntima relación con las causas del mal, cabe puntualizar aquí algunos de los aspectos mencionados en el punto 4 .

\subsection{Profección indirecta}

Para que las armaduras del hormigón estén protegidas al máximo es preciso: respecto del hormigón:

I) que el hormigón sea uniforme y homogéneo;

II) que el hormigón sea compacto y durable. 
Para esto, a su vez, deben cumplirse una serie de condiciones por parte de los materiales, de la dosificación de los mismos, de su puesta en obra, de su curado y de la propia ejecución de la obra o estructura;

respecto de los materiales en general:

III) éstos deben cumplir individualmente con los requisitos necesarios para alejar el peligro de corrosión.

Y para ello ha de verificarse: respecto de los conglomerantes:

IV) que no se mezclen conglomerantes distintos ni se empleen éstos en diferentes partes de una misma obra o estructura;

V) que el cemento no sea expansivo ni retractivo, a fin de que no pueda dar lugar a fisuras por las que penetren agentes corrosivos. Considérese a estos efectos que las microfisuras que algunos tienen por inocuas pueden convertirse en macrifisuras, por lo que siempre constituyen un peligro potencial;

VI) que el cemento sea preferentemente portland, porque los que contienen escorias o puzolanas, o ambos materiales, confieren una menor protección contra la corrosión, aun en ausencia de cloruro cálcico; en base a su menor reserva alcalina. Sin embargo, en estructuras enterradas o sumergidas en las que la corrosión de las armaduras puede ser precedida por una destrucción del propio hormigón, e iniciada y activada por ella, los conglomerantes puzolánicos y siderúrgicos pueden ser utilizados con eficacia, por conferir mayor durabilidad que los portland a la parte pétrea del conglomerado;

respecto de los áridos:

VII) que los áridos no sean porosos ni deleznables;

VIII) que la granulometría de los mismos sea la más adecuada;

IX) que no sean reactivos con los álcalis del cemento y no den lugar a productos expansivos que ocasionen fisuras que a su vez aceleren la corrosión (véase lo indicado en $\mathrm{V}$ );

$\mathrm{X})$ que no contengan sales solubles (arenas de playa, sin lavar);

respecto de las aguas de amasado:

XI) que las aguas de amasado no contengan sales solubles en cantidad peligrosa (aguas de mar);

respecto de las adiciones (si se emplean):

XII) que no contengan cloruro cálcíco, ni otros cloruros o sales que puedan ser de naturaleza corrosiva o favorecedora de la corrosión;

respecto de las armaduras:

XIII) que las armaduras sean de un acero uniforme y de la misma calidad;

XIV) que, en relación con lo precedente, se tenga en cuenta a todos los efectos la clase de acero en relación con la clase de hormigón. Por ejemplo, los aceros estirados para hormigón pretensado son más sensibles a la corrosión que los aceros para hormigón armado corriente, y los aceros suaves se corroen más que los de alta resistencia a la tracción;

XV) que las armaduras tengan la mayor sección posible y nunca un diámetro menor al normal en cada caso (hormigón armado o pretensado); 
XVI) que la superficie de las armaduras sea tal que permita la máxima adherencia al hormigón (corrugados, etc.), pero sin que ello facilite la formación de huecos entre el hormigón y la armadura;

XVII) que el herrumbrado inicial de la superficie de las armaduras, ya que no se pueda eliminar en su totalidad, sea al menos regular y homogéneo. Si no lo es, debe procederse a una limpieza;

XVIII) que la superficie de las armaduras esté exenta de sales producto de corrosión, si han estado expuestas a la intemperie en ambientes agresivos (marítimos, por ejemplo), procediéndose a una limpieza en caso contrario;

XIX) que la cantidad de armaduras sea la adecuada, sin que las haya en un exceso inútil. Para ello se deberá reducir al mínimo posible el número de kilogramos de armadura por metro cúbico de hormigón, y, dentro de ello, reducir la superficie del acero al máximo;

$\mathrm{XX}$ ) que las armaduras tengan los recubrimientos máximos posibles en cualquier parte de la obra, y más aún en las partes más expuestas. El recubrimiento es función de la permeabilidad, pero es preferible un recubrimiento impermeable a un recubrimiento grueso, es decir, que espesor de recubrimiento e impermeabilidad (o calidad del hormigón) son magnitudes conjugadas: si a calidad constante varía el espesor del recubrimiento es como si a espesor de recubrimiento constante variase la impermeabilidad o la calidad del hormigón;

respecto de la dosificación:

XXI) que la dosificación de cemento sea elevada. No parece influir esto en el momento de comienzo de la corrosión, pero, una vez iniciada ésta, se produce con tanto menor velocidad cuanto mayor es la dosificación, por ser mucho menor el coeficiente de permeabilidad del hormigón. La mayor «reserva alcalina» de un hormigón con dosificación elevada de cemento puede tener efecto favorable;

XXII) que la relación agua/cemento no sea ni muy alta ni demasiado baja, debiendo evitarse los morteros secos o muy aguados y los hormigones secos. La mejor consistencia es la plástica. Una vez iniciada la corrosión, ésta se produce con tanto mayor velocidad cuanto mayor es la relación agual cemento, por ser mucho mayor el coeficiente de permeabilidad del hormigón. Además, una mayor cantidad de agua no combinada actúa como vehículo electroquímico y facilita los procesos corrosivos;

respecto de la puesta en obra:

XXIII) que la puesta en obra cumpla lo mejor posible y lo más regular y completamente su cometido;

respecto de la ejecución en general:

XXIV) que la ejecución sea lo más cuidada posible en todos sus detalles;

respecto del curado:

XXV) que el curado del hormigón sea óptimo, debiendo evitarse al máximo las desecaciones superficiales del material en las primeras edades;

XXVI) que, en la medida de lo posible, se eviten las variaciones ambientales de condiciones $\mathrm{y}$, en particular, las alternancias de humedad, sequedad y sa- 
linidad del medio ambiente (mareas, ciclos de calentamiento, enfriamiento y aireación, etc.). Como esto no es en general fácil ni a veces posible, cuando a pesar de todo haya que poner en ambientes agresivos hormigones con armaduras muy superficiales, es imprescindible impermeabilizar las caras externas del hormigón;

XXVII) que se tenga en cuenta la influencia aceleradora de la temperatura en los procesos de corrosión (por ejemplo en hormigones para silos de recogida de cok, etc.);

respecto de las acciones electroliticas:

XXVIII) que se evite al máximo la posibilidad de formación de macroelementos galvánicos por contacto eléctrico de las armaduras con metales diferentes y contacto electrolítico a través del hormigón. La formación de uno de estos macroelementos da lugar a una corrosión mucho más perniciosa, por más intensa y localizada, que muchos microelementos muy repartidos;

XXIX) que se tomen las medidas oportunas para impedir la acción electrolitica por corrientes vagabundas. Tanto en este caso como en el anterior, si en el hormigón existen sales alcalinas (por estar expuesto a condiciones marítimas, por ejemplo) se produce en las zonas catódicas hidróxido sódico que daña al hormigón y destruye la adherencia de éste a las armaduras; esto aparte de las acciones expansivas ya conocidas. Es lo que ocurre en la electrólisis del hormigón con corriente continua, antes de producirse otros efectos mayores por desprendimiento de gases a altas densidades de corriente. Con corriente alterna los efectos no son tan acusados. La corrosión anódica con polarización externa es mucho mayor que la corrosión espontánea, tanto si existen cloruros como si no.

Los aspectos expuestos difícilmente pueden ordenarse por importancia, porque no son independientes, sino que, a veces, son hasta contradictorios, al menos en apariencia. Así, por ejemplo, el grosor del recubrimiento tiene menos importancia con consistencias plásticas del hormigón, o cuando el hormigón es bueno; el grosor de recubrimiento mínimo depende también de la cantidad de cloruro cálcico añadida ạl hormigón y del ambiente, etc.

Por otra parte, si bien es verdad que una dosificación alta de cemento reduce el coeficiente de permeabilidad del hormigón, también es cierto que los hormigones muy altamente dosificados de cemento son más susceptibles de fisuración por retracción y que, sometidos a esfuerzos de tracción, las fisuras pueden acentuarse y contribuir a acelerar o provocar la corrosión. Además, un hormigón muy rico en cemento, pero con un contenido mayor de cloruro cálcico puede experimentar mayor corrosión en sus armaduras que otro menos rico, pero también con menos cloruro cálcico. Es decir, no siempre se contrarrestan los efectos, y por ello existen límites para la influencia ventajosa o perjudicial, de los aspectos considerados. Por ejemplo, parece ser también que la corrosión debida al cloruro cálcico de algunos productos de adición para el hormigón, de acción aireante o fluidificante, es de escasa importancia.

De otro lado, hay opiniones según las cuales la formación de cloroaluminatos entre el aluminato tricálcico del cemento portland y el cloruro cálcico, apenas influye en la reducción de la corrosión debida a este último. Con carácter más general puede admitirse, en cambio, que son menores los peligros por reducción de la sección de las armaduras y consiguiente disminución de su capacidad de resistencia a la tracción, que por pérdida de adherencia entre ellas y el hormigón.

Lo indicado por vía de ejemplos viene a corroborar la idea de que es necesario justipreciar todos los aspectos de la corrosión de armaduras con criterio ponderado, pues en otro caso es muy fácil encontrar contradicciones y caer en juicios erróneos al valorar los hechos. 


\subsubsection{El problema de la fisuración}

Se ha mencionado varias veces en lo que precede el tema de las fisuras, interesante hasta el punto de que se ha querido poco menos que atribuir a él en exclusiva la causa de la corrosión, y en tal sentido se ha tratado de señalar un tamaño crítico de fisura, o más todavía, dos tamaños críticos, para medios más y menos agresivos, por debajo de cuyos tamaños no se produce corrosión.

No es fácil definir el tamaño de la fisura, pues éste depende de que se mida en la superficie del hormigón o se calcule a partir de esta medida el valor correspondiente a su ancho en la zona adyacente a la armadura, lo cual parece más lógico.

La impresión general es que no existe tal tamaño crítico y que tan importantes como el ancho de las fisuras son otros factores que se relacionan con dicho ancho (de lo cual puede nacer el equívoco).

Lo innegable es, por una parte, que las fisuras pequeñas pueden agrandarse, por lo que constituyen un peligro potencial de corrosión y, por otra, que cuanto menor es el ancho de las fisuras, menor es la velocidad con que la corrosión procede.

Tampoco debe subestimarse el hecho de que entre corrosión y fisuración existe una relación de causa o efecto no siempre bien definida en cuanto a prioridad, puesto que la fisuración puede dar lugar a corrosiones que sin ella no se producirían, o lo harían menos rápida e intensamente, y la corrosión puede causar fisuraciones que la faciliten e incrementen de forma notoria.

Todo fenómeno, acción o circunstancia que tienda a producir o incrementar la fisuración, tiende a producir o incrementar también la corrosión.

\subsection{Protección directa}

Aparte de los remedios expuestos en 7.2, a los que cabe considerar como de protección indirecta, que a pesar de lo cual son los más importantes y que, como se ha podido apreciar están de la mano del técnico del hormigón, deben tenerse en cuenta los que en antítesis constituyen una protección directa y son del dominio del técnico de la corrosión. Estos consisten en:

I) emplear recubrimientos impermeabilizantes protectores del hormigón, como se ha indicado en 7.2, XXVI), y procurar al máximo la pasividad natural del metal, pues su mejor protección la da su estado de pasividad total. Para ello:

II) añadir inhibidores de corrosión a la masa del hormigón que den a la superficie del acero una pasividad verdadera y un seudo-potencial suficientemente regular y fijo, situado entre los valores correspondientes a los potenciales de oxidación y a los de disolución. Estos inhibidores suelen ser fluoruros (simples o complejos: fluosilicatos y fluoaluminatos) y fosfatos (simples o dobles, alcalinos o alcalinotérreos) poco solubles en medio alcalino de cal (en el que el hierro no se corroe) y más solubles en medios ácidos y salinos corrosivos. Los inhibidores en estado pulverulento muy fino y en proporciones muy pequeñas se añaden al hormigón, sin que en general perturben al cemento (los fluoruros en grandes dosis son retardadores del fraguado y del endurecimiento). Lo que se puede conseguir así es el retraso o la evitación de los primeros desequilibrios locales del potencial y, en consecuencia, soslayar a poco precio pequeños riesgos que frecuentemente podrían degenerar en otros mucho más serios. La acción de estos inhibidores suele tener su importancia, sobre todo en el caso de que en el hormigón existan cloruros y haya polarización externa. Con un inhibidor de tipo fosfato, por ejemplo, puede conseguirse que la corrosión anódica en presencia de cloruros se reduzca a la que se tendría en 
ausencia de los mismos. De todos modos, los inhibidores no parecen resolver los problemas de corrosión realmente graves;

III) prever en el proyecto de la obra de hormigón armado, cuando las circunstancias lo exijan, un sistema de protección catódica, a ser posible desde el primer momento. La protección catódica, tanto si se consigue mediante ánodos de sacrificio, como si responde a la acción de una corriente exterior aplicada, para ser eficaz y rentable ha de ser bien estudiada y vigilada. En el primer caso, para la reposición de ánodos a su debido tiempo y, en el segundo, para que la corriente sea lo suficientemente intensa e ininterrumpida, o actúe durante el tiempo suficiente para garantizar el alejamiento de los iones salinos de la zona catódica protegida, creando al interrumpirse un potencial pasivo $y$ estable en el acero. De no ser así, es un procedimiento preventivo caro y no $\tan$ eficaz como debería serlo, en correspondencia a su costo. Sin embargo, cuando se aplica conscientemente da unos excelentes resultados, como se ha puesto de manifiesto en una gran red de regadío construida en Argelia, en la que se ha instalado un sistema de protección que, al parecer, es el primero del mundo que se ha manifestado como verdaderamente eficaz.

\section{Bomctusiones}

De cuanto queda expuesto pueden inferirse las siguientes conclusiones:

I) la corrosión de las armaduras, como fenómeno químico o electroquímico que puede afectar a los hormigones armados y pretensados, es un aspecto importante en la durabilidad de estos materiales y de los elementos o estructuras a que dan lugar;

II) la corrosión de las armaduras es un riesgo potencial de actualización más o menos probable según las circunstancias y las medidas que se adopten para evitarlo;

III) las medidas y precauciones constituyen un seguro contra el citado riesgo. Este seguro, como todos, es una "medicación" preventiva; la "póliza" del seguro debe concertarse, o el "medicamento" tomarse, antes de que se produzca el siniestro o se presente el mal. La solución del problema de corrosión es entonces siempre posible, y casi siempre económica, sobre todo con miras a los resultados a largo plazo. Los remedios curativos «a posteriori», cuando el daño se ha producido, si los hay, no siempre son fáciles ni posibles de aplicar, y casi siempre son muy costosos;

IV) los problemas de corrosión de armaduras del hormigón se plantean siempre en un terreno común a la Electroquímica y a la Tecnología de la Construcción y de sus Materiales, por lo que son de la competencia de técnicos de ambas especialidades. En circunstancias en que el técnico de la construcción tenga problemas o crea tenerlos, en relación con la corrosión metálica, y no pueda resolverlos por sí mismo, debe consultar con el especialista en corrosión, ya desde la etapa de proyecto, y antes de proceder a la ejecución. A tal efecto van siendo cada vez más numerosas las casas y firmas dedicadas a tratamientos y protección anticorrosivos, con su personal técnico especializado, al servicio de los usuarios;

V) la repercusión no ya técnica, sino económica, de los problemas de corrosión es enorme, sin contar con las consecuencias de tipo legal y de responsabilidad a que dichos problemas pueden dar lugar;

VI) como toda materia con una doble vertiente científica y técnica, la corrosión de armaduras en el hormigón requiere, para su evitación y la de sus consecuencias, conocimiento y bien obrar. 


\section{Fillshiogureaffia}

BERTHIER, R. M.: La corrosion du fer en général et dans le cas particulier des armatures du béton. «Revue des Matériaux de Construction», núm. 511, 101-105 (1951).

Brocard, J.: Corrosion des aciers dans le béton armé. "Annales de l'Institut Téchnique du Bâtiment et des Travaux Publics», núm. 126, junio 1958.

Calleja, J.: Influencia del contenido de yeso del cemento portland sobre la retracción de las pastas, morteros y hormigones. Trabajos de la III Reunión Internacional sobre Reactividad de los Sólidos. Sección V, Madrid, 1956.

- El cloruro cálcico en la Construcción (Manual). «Manuales y Normas del I.E.T.c.c.», Madrid, 1957.

- El cloruro cálcico como acelerador en la prefabricación de hormigón. «Monografía núm. 185 del I.E.T.c.c.», Madrid, 1958; "Revista de Ciencia Aplicada» XII, 122 (1958); "Ultimos Avances en Materiales de Construcción» (I.E.T.c.c.), núm. 86 (1958).

COPEnHagen, W. J.: Corrosion and protection of metals used in the Building Industry. "National Chemical Research Laboratory», Cape Town.

- Sulphur as a factor in the corrosion of iron and steel structures in the sea. "Transactions Royal Society of South Africa», 22, 2 (1954). South African Industrial Chemist, febrero 1954.

HrLl, A. W.: La corrosion des aciers dans le béton contenant du chlorure de calcium. "IV Congrès International du Béton Manufacturé», París, 1963.

Lewis, D. A., y CoPENHAgen, W. J.: The corrosion of reinforcing steel in marine atmospheres. "South African Industrial Chemist», 11-10 (1957).

LOBRY DE BRUIN, C. A.: Influence of cracking on corrosion of reinforcement. "General report. Parte IV (a) de "Cracks in concrete and corrosion of steel reinforcing bars». RILEM-Symposium, Stockholm, 1957.

Maraghini, M., y Ginnari, C.: Corrosione anodica del ferro nel cemento armato. "La Ricerca Scientifica», 27-5, 1500-1508 (1957).

MaRAghint, M., y Rumi, B.: Misure elettrochimici su provini d'acciaio inmersi nel cemento. "La Ricerca Scientifica», 27, 1161-1174 (1957).

Moldovan, V.: Recherches sur la corrosion des armatures dans les bétons soumis à des actions aggresives. "Revue des Matériaux de Construction», núm. 570, 98-99 (1963).

MOLE, G.: The effects of electrolysis upon the strength of reinforced concrete. "The Technical Electrical and Allied Industries Research Association». Technical Report O/T3, London, 1948.

MONFORE, G. E., y VERBECK, G. J.: Corrosion of prestressed wire in concrete, "Journal of the American Concrete Institute», 32-5, 493-515 (1960).

Nicol, L.: Observations et remarques sur le potentiel de contact fer-ciment. "Revue des Matériaux de Construction», núms. 487 (71-82), 44 (103-108), 489 (133-140), 1956.

OST, B., y MONFoRE, G. E.: Corrodibility of prestressing wire in concretes made with type $I$ and type IS cements. "Portland Cement Association. Research Dept.», Bull. 159. Skokie, Illinois, 1963.

REINSDORF, S., y Riston, P.: Untersuchungen über die Korrosion des Bewehrunsstahles bei Verwendung von Kalziumchlorid im Mörtel und Beton. "Silikattechnik, 11-10, 478-483 (1960).

ROBERTS, M. H.: Effect of calcium chloride on the durability of pre-tensioned wire in prestressed concrete. "Magazine of Concrete Research", 14-42, 143-154 (1962).

Sersale, R., y Frigione, G.: I solfuri presenti nel cemento e la resistenza alla corrosione dei ferri delle armature. «L'Industria Italiana del Cemento", 33 (9), 687 (1963).

WALZ, K.: Corrosion of prestressing steel in concrete made of blast-furnace cement. CEMBUREAU, Annex to Circular letter 49. HC/ps (confidential), Malmö, 1963.

AUTORES VARIOS: La corrosión y su control. Boletín número 11 del Colegio Oficial de Doctores y Licenciados en Ciencias Químicas y Físico-Químicas. Madrid, noviembre 1963. 


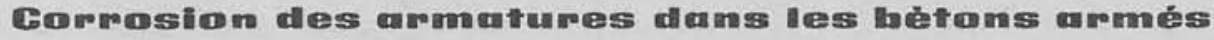

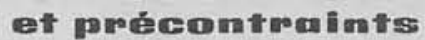

\author{
Dr. J. Calleja
}

Dans le chapitre général consacré à la «durabilité» ou résistance chimique dans le temps des liants hydrauliques (mortiers et bétons armés et précontraints), la partie concernant la «corrosion» des armatures occupe une place prépondérante pour son importance technique et économique.

Cette importance est reconnue de plus en plus, bien que la corrosion soit (ou ait été) un des thèmes les moins familiers dans le do. Cette importance est reconnue de plus en plus, bien que la corrosion soit (ou ait été) un des thèmes les moins familiers dans le do-
maine de la construction. La raison est due à la force des faits et se manifeste nettement dans les tâches confiées et dans les problèmes maine de la construction. La raison est due à la force des faits
posés aux laboratoires de recherche et d'essais des matériaux.

La corrosion est un phénomène physico-chimique qui tombe dans le comaine de l'ELECTROCHIMIE et qui doit être étudié et jugé conformément aux lois fondamentales de cette discipline. II en est de même pour ce qui est des méthodes de «protection» contre la corrosion.

La corrosion est un «risque» aléatoire que l'on court et contre lequel il faut se garder à l'aide d'une assurance, représentée par ces mesures de protection.

Le remède ale plus facile» de point de vue «technique», et «moins coûteux» du point de vue «économique» est toujours de caractère "préventif» plutôt» que «curatifn, c'est-â-dire celui qui a été pris à l'étape du projet de l'ouvrage ou de la structure dont il s'agit. Dans le cas du béton, la meilleure protection des armatures est donnée, en premier lieu, par l'application d'un «code de bonne pratique»
à l'élaboration du matériau at à l'exécution de l'ouvrage, et, en second lieu, par l'observance des principes les plus élémentaires de l'électrochimie en ce qui concerne la corrosion.

L'utilisation d'additifs accélérants de prise et de durcissement de nature «chloruréen et l'état de "contrainte» des armatures, sont des aspects qui méritent une consider
les armatures de la structure.

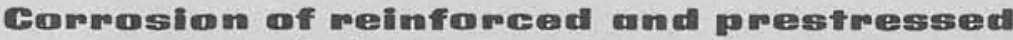

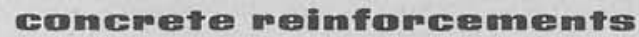

Dr. J. Calleja

Within the general question of «durability», or chemical resistence of hydraulic cements in the course of time (mortars and reinforced and prestressed coneretes), the problem of reinforcement ecorrosions is most important because of its technical and economic significance. This importante is being increasingly appreciated every day, even though corrosion is (or has been) one of the least well known subjects within the field of construction. This importance is evident from the facts themselves, which become clear as a result of the problems that the research laboratories and testing establishments have to deal with.

Corrosion is a physicochemical phenomenon which belongs to the ambit of ELECTROCHEMISTRY, and should be studied and evaluated in accordance with the fundamental laws of electrochemistry itself. The same applies to the methods of protection that are to be adopted against corrosion.

Corrosion is a chance «risk», and one should seek protection against it by some form of «insurance», represented by the measures to avoid it.

The «easiest» cure, from a «technical» point of view, and the «cheapest» from the «economical» aspect, is always a «preventive» one, rather than a ccurative one, $\mathbf{i}$. e., the measures of protection should be taken at the design stage of the structure or work in question. In the case of concrete, the best protection for reinforcements is, in the first place, the adoption of a code of egood practices in the
ellaboration of the material and the workmanship, and in the second place, the observance of the most elementary electrochemical principles in the actual matter of corrosion.

The use of accelerating additives in the setting and hardening which are of «chlorinated» nature, and the state of «stress» of the reinforcements, are aspects which deserve special interest, as well as the possible electrochemical actions due to *stray currents» travelling cements, are aspects which deserve special inte
through the reinforcements of the structure.

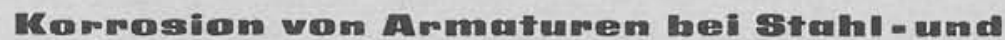 Morspanmibetem}

Dr. J. Calleja

Im allgemeinen Kapitel über «Dauerhaftigkeit» oder chemische Beständigkeit der hyđraulischen Konglomerate (Stahlmörteln und Stahl und Vorspannbeton) nimmmt der Abschnitt über «Korrosions der Armaturen einen hervorragenden Platz ein wegen seiner technischen und wirtschaftlichen Bedeutung.

Diese Bedeutung tritt jeden Tag mehr zutage, obwohl die Korrosion eines der am wenigsten vertrauten Kapitel im Rahmen des Bauwesens ist (oder gewesen ist). Der Grund liegt in der Gewalt der Tatsachen und äussert sich klar in den den Materialforschung. und Prüflaboratorien übertragenen und unterbreiteten Problemen.

Die Korrosion ist ein physikalisch-chemischer Vorgang, der zu dem Gebiet der ELEKTROCHEMIE gehört und der gemäss den grundlegenden Gesetzen dieser Dizsiplin untersucht und beurteilt werden muss. Dasselbe gilt für die $\alpha$ Schutzmethoden» gegen Korrosion.

Die Korrosion ist ein auf Zufall beruhendes $\alpha$ Risiko», das man eingeht und gegen welches man sich mittels einer «Versicherung» in Form dieser Schutzmassnahmen vorsehen muss.

Das «leichteste» Mittel vom «technischen» Gesichtspunkt und das «billigste» vom «wirtschaftlichen» Standpunkt aus ist immer das «vorbeugende» und nicht das «abhelfende», das heisst, ein Mittel, das schon in der Etappe der Projektierung des Baus oder der Struktur, um die es sich handelt, vorgesehen wurde.

Im Falle des Betons besteht der beste Schutz der Armaturen in erster Linie in der Anwendung eines «Kodex über gesunde Praxis» bei der Herstellung des Materials und der Ausführung des Baus und in zweiter Linie in der Berüicksichtigung der elementarsten Begriffe der Elektrochemie

Die Verwendung von «chloridhaltigen» Zusätzen zur Beschleunigung der Bindung und Erhärtung, und der «Spannungszustand» der Arma. turen sind Gesichtspunkte, die einer besonderen Beachtung bedürfen, sowie die möglichen elektrochemischen Einwirkungen zufolge der «ableitenden Ströme» in den Armaturen der Struktur. 